\title{
A Novel Data-Driven Terminal Iterative Learning Control with Iteration Prediction Algorithm for a Class of Discrete-Time Nonlinear Systems
}

\author{
Shangtai Jin, ${ }^{1}$ Zhongsheng Hou, ${ }^{1}$ and Ronghu $\mathrm{Chi}^{2}$ \\ ${ }^{1}$ Advanced Control Systems Lab, School of Electronic \& Information Engineering, Beijing Jiaotong University, Beijing 100044, China \\ ${ }^{2}$ School of Automation \& Electronic Engineering, Qingdao University of Science \& Technology, Qingdao 266042, China \\ Correspondence should be addressed to Shangtai Jin; shtjin@bjtu.edu.cn
}

Received 15 May 2014; Accepted 23 July 2014; Published 12 August 2014

Academic Editor: Claudio H. Morales

Copyright (C) 2014 Shangtai Jin et al. This is an open access article distributed under the Creative Commons Attribution License, which permits unrestricted use, distribution, and reproduction in any medium, provided the original work is properly cited.

A data-driven predictive terminal iterative learning control (DDPTILC) approach is proposed for discrete-time nonlinear systems with terminal tracking tasks, where only the terminal output tracking error instead of entire output trajectory tracking error is available. The proposed DDPTILC scheme consists of an iterative learning control law, an iterative parameter estimation law, and an iterative parameter prediction law. If the partial derivative of the controlled system with respect to control input is bounded, then the proposed control approach guarantees the terminal tracking error convergence. Furthermore, the control performance is improved by using more information of predictive terminal outputs, which are predicted along the iteration axis and used to update the control law and estimation law. Rigorous analysis shows the monotonic convergence and bounded input and bounded output (BIBO) stability of the DDPTILC. In addition, extensive simulations are provided to show the applicability and effectiveness of the proposed approach.

\section{Introduction}

Iterative learning control (ILC) is able to refine the control signals at current iteration by utilizing the input and output (I/O) data of previous iterative operations. As a direct result, the tracking error accuracy is improved as the number of repetitions increases. ILC has attracted much attention in the past three decades due to its simplicity and efficiency [1-3].

In practice, the ultimate control objective for many practical plants, such as rapid thermal processing systems for chemical vapor deposition (RTPCVD) [4], thermoforming ovens [5], and station stop control of a train [6], is the terminal state or terminal output instead of the entire trajectory of the system output. And the only measurement available for some plants $[4,5]$ is the terminal state or terminal output. For such a control task, the conventional ILC methods, which need to handle the desired output trajectory in a given time interval, are not applicable because the exact measurements of all the system states or outputs over the entire finite time interval are impossible [4] or unnecessary [6].

To overcome this problem, terminal iterative learning control (TILC) [4-6] and point-to-point iterative learning control (PTP-ILC) [7-9] are derived from ILC theory to use intermediate point or terminal point of every run. Recently, PTP-ILC and TILC are becoming a new research direction of ILC both in theory and in practical applications. The existing research results of PTP-ILC and TILC mainly focus on contraction-mapping-based learning law $[4,6]$ and optimization-based learning law [7-11].

Although the contraction-mapping-based TILC methods in $[4,6]$ are applicable for nonlinear systems, the proper selection of the learning gain is not a trivial thing in practical applications when there is little prior knowledge about the controlled system. Another limitation is that the TILC schemes proposed in [6] fix the learning gain through all iterations without any tuning and thus lack flexibility and 
adaptability regarding the expansions of the controlled plant and the exogenous uncertainties.

The optimal TILC $[10,11]$, where the explicit optimal cost function is given and minimized to design optimizationbased TILC algorithm, can guarantee monotonic convergence along the iteration axis. However, it depends on the knowledge of a perfect model. If there is a lack of an accurate model, the monotonic convergence is no longer guaranteed.

As we know, the scale of many industrial processes, such as chemical industry, metallurgy, machinery, and transportation, becomes increasingly large, and the production technology and processes also become more and more complex. As a direct result, modeling these processes by using the first principles or identification methods becomes more and more difficult. Apparently, it will meet many limitations in practice when applying the conventional model-based control approaches. On the other hand, however, many industrial processes generate and store a huge amount of process data containing some valuable state information of the process operations and the equipment $[12,13]$, which motivates us to study the data-driven control methods.

More recently, a general data-driven optimal terminal iterative learning control approach, which is available for both linear and nonlinear systems, is proposed in [14]. Only the updated control input and the measured system output at the terminal point are utilized for the controller design and analysis. However, it is noted that the proposed approach in [14] is only of one-order with respect to the control input and the terminal tracking error. That is, only the I/O data of the previous one iteration is used in that control approach [14], which may reduce its robustness to iteration-dependent disturbances and uncertainties in practical applications.

Model predictive control (MPC) has been introduced to enhance the robustness of the ILC design $[15,16]$ by using the predictive $\mathrm{I} / \mathrm{O}$ data within a prespecified time horizon. It is obvious that the more information is exploited, the more flexible the controller design and the better control performance may become. However, similar to the optimal ILC methods, the predictive ILC $[15,16]$ also requires that the controlled plant is an exact known linear system, or, at least, an approximate linear model of the controlled plant is known a priori.

In this work, a data-driven predictive TILC (DDPTILC) scheme is proposed by combining the advantages of datadriven optimal TILC [14] and predictive control [17-19]. An equivalent linear iteration-varying data model is developed first for the repeatable nonlinear system with a terminal tracking task. And then, the DDPTILC scheme is designed based on the equivalent data model. The control scheme consists of an iterative learning control law, a parameter iterative estimation law, and a parameter iterative prediction law and is updated iteratively by using the I/O data only. In more detail, the DDPTILC first estimates and predicts the partial derivatives of the controlled plant with respect to control inputs in the iteration domain and then uses the equivalent data model with estimated partial derivatives to predict the terminal output within a prespecified prediction iteration horizon; finally, it calculates the optimal control sequence by minimizing a given objective function.
The proposed approach is a kind of data-driven control method, since the controller design requires only the measured I/O data. Only the information about the lower and upper bounds of the partial derivative of the nonlinear discrete-time system with respect to control input is needed to analyze the bounded input and bounded output (BIBO) stability and terminal tracking error convergence. Numerical simulations show that the proposed approach contributes a better control performance and robustness by using the predictive information within a prespecified iteration horizon.

The rest of this paper is organized as follows. Section 2 is the problem formulation. The DDPTILC scheme is designed and analyzed in Section 3. Section 4 shows the monotonic convergence of the DDPTILC scheme. Section 5 provides numerical simulations to show the effectiveness of the DDPTILC scheme. Finally, some conclusions are given in Section 6.

\section{Problem Formulation}

Consider the following SISO discrete-time nonlinear system:

$$
y_{i}(k+1)=f\left(y_{i}(k), u_{i}(k)\right)
$$

where $k=0,1, \ldots, N$ is the sampling time index; $N$ is the finite time interval of the run-to-run system; $i$ denotes the system repetition number; $y_{i}(k) \in R$ is the system output, where only $y_{i}(N)$ is measurable at the end of every run; $u_{i}(k) \in R$ denotes the control input, which is time-invariant at all sampling time in the same run, and $f(\cdots)$ is an unknown scalar nonlinear function and continuously differentiable.

The relationship between the input and output sequences can be expressed by the following equations:

$$
\begin{aligned}
y_{i}(1) & =f\left(y_{i}(0), u_{i}\right)=g_{1}\left(y_{i}(0), u_{i}\right), \\
y_{i}(2) & =f\left(y_{i}(1), u_{i}\right)=f\left(g_{1}\left(y_{i}(0), u_{i}\right), u_{i}\right) \\
& =g_{2}\left(y_{i}(0), u_{i}\right), \\
& \vdots \\
y_{i}(N) & =f\left(y_{i}(N-1), u_{i}\right)=f\left(g_{N-1}\left(y_{i}(0), u_{i}\right), u_{i}\right) \\
& =g_{N}\left(y_{i}(0), u_{i}\right),
\end{aligned}
$$

where $y_{i}(0)$ is the initial value of system $(1)$, and $g_{1}(\cdots)$, $g_{2}(\cdots), \ldots, g_{N}(\cdots)$ are the corresponding nonlinear functions and differentiable to all the arguments.

Define the terminal output difference between consecutive two iterations as

$$
\Delta y_{i}(N)=y_{i}(N)-y_{i-1}(N)
$$

Using (2) and mean value theorem, (3) is rewritten as

$$
\begin{aligned}
\Delta y_{i}(N) & =g_{N}\left(y_{i}(0), u_{i}\right)-g_{N}\left(y_{i-1}(0), u_{i-1}\right) \\
& =\frac{\partial g_{N}^{*}}{\partial y}\left(\xi_{i}\right) \Delta y_{i}(0)+\frac{\partial g_{N}^{*}}{\partial u}\left(\zeta_{i}\right) \Delta u_{i},
\end{aligned}
$$

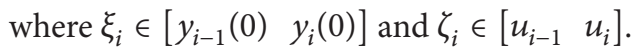


Two assumptions are exposed on system (1) to restrict our discussion.

Assumption 1. The initial value $y_{i}(0)$ is identical for every iteration; that is, $y_{i}(0)=$ const., $i=1,2, \ldots$.

Assumption 2. $\partial g_{N}^{*} / \partial u$ has lower bound $\alpha_{1}$ and upper bound $\alpha_{2}$, and $0<\alpha_{1}<\alpha_{2}$ or $\alpha_{2}<\alpha_{1}<0$. Without loss of generality, we only discuss the case of $0<\alpha_{1}<\alpha_{2}$ in this paper.

In terms of Assumption 1, (4) becomes

$$
\Delta y_{i}(N)=\frac{\partial g_{N}^{*}}{\partial u}\left(\zeta_{i}\right) \Delta u_{i}=\theta_{i} \Delta u_{i}
$$

where $\theta_{i}=\left(\partial g_{N}^{*} / \partial u\right)\left(\zeta_{i}\right)$, and $\alpha_{1} \leq \theta_{i} \leq \alpha_{2}$.

Based on (5), the terminal output prediction equation is given as

$$
\begin{aligned}
y_{i}(N) & =y_{i-1}(N)+\theta_{i} \Delta u_{i}, \\
y_{i+1}(N) & =y_{i}(N)+\theta_{i+1} \Delta u_{i+1} \\
& =y_{i-1}(N)+\theta_{i} \Delta u_{i}+\theta_{i+1} \Delta u_{i+1}, \\
\vdots & \\
y_{i+N_{y}-1}(N) & =y_{i+N_{y}-2}(N)+\theta_{i+N_{y}-1} \Delta u_{i+N_{y}-1} \\
& =y_{i-1}(N)+\theta_{i} \Delta u_{i}+\theta_{i+1} \Delta u_{i+1} \\
& \quad+\cdots+\theta_{i+N_{y}-1} \Delta u_{i+N_{y}-1} .
\end{aligned}
$$

Let

$$
\begin{gathered}
\mathbf{Y}_{i}=\left[y_{i}(N), \ldots, y_{i+N_{y}-1}(N)\right]^{T}, \\
\Delta \mathbf{U}_{i}=\left[\Delta u_{i}, \ldots, \Delta u_{i+N_{y}-1}\right]^{T}, \quad E=[1,1, \ldots, 1]^{T}, \\
\mathbf{A}_{i}=\left[\begin{array}{ccccc}
\theta_{i} & 0 & \ldots & & 0 \\
\theta_{i} & \theta_{i+1} & 0 & \ldots & 0 \\
& \theta_{i+1} & \ddots & \ddots & \vdots \\
\vdots & \vdots & \ddots & \ddots & 0 \\
\theta_{i} & \theta_{i+1} & & \theta_{i+N_{y}-2} & \theta_{i+N_{y}-1}
\end{array}\right],
\end{gathered}
$$

where $\mathbf{Y}_{i}$ and $\Delta \mathbf{U}_{i}$ denote the prediction vector of the terminal output and predictive control input increment vector at iteration $i$, respectively. $N_{y}$ is the prediction horizon.

Equation (6) can be rewritten in a compact form:

$$
\mathbf{Y}_{i}=E y_{i-1}(N)+\mathbf{A}_{i} \Delta \mathbf{U}_{i} .
$$

Remark 3. It is noted that the prediction equation (8) is obtained from (5), which constructs an iteration-related linear relationship of control input and terminal output of the original nonlinear system (1). It is a completely equivalent transformation without any omitting, such as higher-order terms.

The control objective is to track a given desired output signal $y_{i}^{*}(N)$ at the single terminal point by generating an optimal control signal $u_{i}^{*}$.

\section{Data-Driven Predictive Terminal ILC Design}

Consider the following cost function of control input:

$$
J=\sum_{j=0}^{N_{y}-1}\left[\left(y_{i}^{*}(N)-y_{i+j}(N)\right)^{2}+\lambda \Delta u_{i+j}^{2}\right],
$$

where $\lambda>0$ is a weighting factor, and $y^{*}(N)$ is the desired terminal output.

Remark 4. Note that $\lambda$ is an important parameter. The proper selection of $\lambda$ can guarantee the stability and improve the tracking performance.

Let $\mathbf{Y}_{i}^{*}=\left[y_{i}^{*}(N), \ldots, y_{i+N_{y}-1}^{*}(N)\right]^{T}$; the cost function (9) becomes

$$
J=\left[\mathbf{Y}_{i}^{*}-\mathbf{Y}_{i}\right]^{T}\left[\mathbf{Y}_{i}^{*}-\mathbf{Y}_{i}\right]+\lambda \Delta \mathbf{U}_{i}^{T} \Delta \mathbf{U}_{i} .
$$

Substituting (8) into (10) and using the optimality condition $\partial J / \partial \mathbf{U}_{i}=0$ yield the control law

$$
\Delta \mathbf{U}_{i}=\left[\mathbf{A}_{i}^{T} \mathbf{A}_{i}+\lambda \mathbf{I}\right]^{-1} \mathbf{A}_{i}^{T}\left[\mathbf{Y}_{i}^{*}-E y_{i-1}(N)\right],
$$

where I denotes the unit matrix.

According to the receding horizon principle, the control input at current iteration $i$ is constructed as

$$
u_{i}=u_{i-1}+\mathbf{g}^{T} \Delta \mathbf{U}_{i}
$$

where $\mathbf{g}=[1,0, \ldots, 0]^{T}$.

When $N_{y}=1$, (12) becomes

$$
u_{i}=u_{i-1}+\frac{\theta_{i}\left(y_{i}^{*}(N)-y_{i-1}(N)\right)}{\theta_{i}^{2}+\lambda},
$$

which is same as the control law in [20].

Since $\mathbf{A}_{i}$ in (12) contains unknown parameters $\theta_{i}, \theta_{i+1}$, and $\theta_{i+N_{y}-1}$, the parameter estimation algorithm and prediction algorithm should be developed. Here, the cost function of parameter $\theta_{i}$ is proposed as follows:

$$
J\left(\theta_{i}\right)=\left|y_{i-1}(N)-y_{i-2}(N)-\theta_{i} \Delta u_{i-1}\right|^{2}+\mu\left|\theta_{i}-\widehat{\theta}_{i-1}\right|^{2},
$$

where $\mu>0$ is a weighting factor.

Minimizing (14) with respect to $\theta_{i}$ gives the following projection estimation algorithm:

$$
\widehat{\theta}_{i}=\widehat{\theta}_{i-1}+\frac{\eta \Delta u_{i-1}}{\mu+\Delta u_{i-1}^{2}}\left[\Delta y_{i-1}(N)-\widehat{\theta}_{i-1} \Delta u_{i-1}\right],
$$

where $\mu>0$ is a weighting factor, and $0<\eta \leq 1$ is a step size factor.

The other parameters $\widehat{\theta}_{i+1}, \ldots, \widehat{\theta}_{i+N_{y}-1}$ cannot be directly calculated from $\mathrm{I} / \mathrm{O}$ data till iteration $i$ and thus need to be predicted by certain prediction algorithm. There exist many prediction methods, such as the Aström prediction method [21], the self-tuning method [22], and the multilevel 
hierarchical forecasting method $[23,24]$. According to the simulation results in $[23,24]$, the multilevel hierarchical forecasting method possesses the best predictive error. Thus, the multilevel hierarchical forecasting method $[23,24]$ is applied here to predict the unknown parameters.

Assume that the estimated values $\widehat{\theta}_{1}, \ldots, \widehat{\theta}_{i}$ have been calculated by (15) till iteration $i$. Using these estimated values, an autoregressive (AR) model for prediction is constructed as

$$
\widehat{\theta}_{i+1}=\gamma_{1, i} \widehat{\theta}_{i}+\gamma_{2, i} \widehat{\theta}_{i-1}+\cdots+\gamma_{n_{p}, i} \widehat{\theta}_{i-n_{p}+1},
$$

where $\gamma_{1, i}, \ldots, \gamma_{n_{p}, i}$ are coefficients, and $n_{p}$ is the model order, which is usually set to be $2 \sim 7[23,24]$.

Using (16), prediction equation becomes

$$
\widehat{\theta}_{i+j}=\gamma_{1, i} \widehat{\theta}_{i+j-1}+\gamma_{2, i} \widehat{\theta}_{i+j-2}+\cdots+\gamma_{n_{p}, i} \widehat{\theta}_{i+j-n_{p}},
$$

where $j=1, \ldots, N_{y}-1$.

Define $\boldsymbol{\gamma}_{i}=\left[\gamma_{1, i}, \ldots, \gamma_{n_{p}, i}\right]^{T}$ and $\widehat{\boldsymbol{\theta}}_{i-1}=\left[\widehat{\theta}_{i-1}, \ldots, \widehat{\theta}_{i-n_{p}}\right]^{T}$. $\gamma_{i}$ are determined by following equation:

$$
\boldsymbol{\gamma}_{i}=\boldsymbol{\gamma}_{i-1}+\frac{\widehat{\boldsymbol{\theta}}_{i-1}}{\delta+\left\|\widehat{\boldsymbol{\theta}}_{i-1}\right\|^{2}}\left[\widehat{\theta}_{i}-\boldsymbol{\gamma}_{i-1}{ }^{T} \widehat{\boldsymbol{\theta}}_{i-1}\right] \text {, }
$$

where $\delta \in(0,1]$ is a positive constant.

By integrating the control algorithm (12), the parameter estimation algorithm (15), and the prediction algorithm (17)(18), the data-driven predictive terminal iterative learning control scheme is constructed as follows:

$$
\begin{aligned}
& \widehat{\theta}_{i}=\widehat{\theta}_{i-1}+\frac{\eta \Delta u_{i-1}}{\mu+\Delta u_{i-1}^{2}}\left[\Delta y_{i-1}(N)-\widehat{\theta}_{i-1} \Delta u_{i-1}\right] \text {, } \\
& \widehat{\theta}_{i}=\widehat{\theta}_{1}, \quad \text { if }\left|\hat{\theta}_{i}\right| \leq \varepsilon \text {, or }\left|\Delta u_{i-1}\right| \leq \varepsilon \\
& \text { or } \operatorname{sign}\left(\widehat{\theta}_{i}\right) \neq \operatorname{sign}\left(\widehat{\theta}_{1}\right) \text {, } \\
& \boldsymbol{\gamma}_{i}=\boldsymbol{\gamma}_{i-1}+\frac{\widehat{\boldsymbol{\theta}}_{i-1}}{\delta+\left\|\widehat{\boldsymbol{\theta}}_{i-1}\right\|^{2}}\left[\widehat{\theta}_{i}-\gamma_{i-1}{ }^{T} \widehat{\boldsymbol{\theta}}_{i-1}\right] \text {, } \\
& \gamma_{i}=\gamma_{1}, \quad \text { if }\left\|\gamma_{i}\right\| \geq M \text {, } \\
& \widehat{\theta}_{i+j}=\gamma_{1, i} \widehat{\theta}_{i+j-1}+\gamma_{2, i} \widehat{\theta}_{i+j-2}+\cdots+\gamma_{n_{p}, i} \widehat{\theta}_{i+j-n_{p}} \\
& j=1,2, \ldots, N_{y}-1 \text {, } \\
& \widehat{\theta}_{i+j}=\widehat{\theta}_{1} \text {, if }\left|\widehat{\theta}_{i+j}\right| \leq \varepsilon \text { or } \operatorname{sign}\left(\widehat{\theta}_{i+j}\right) \neq \operatorname{sign}\left(\widehat{\theta}_{1}\right), \\
& j=1,2, \ldots, N_{y}-1, \\
& \Delta \mathbf{U}_{i}=\left[\widehat{\mathbf{A}}_{i}^{T} \widehat{\mathbf{A}}_{i}+\lambda \mathbf{I}\right]^{-1} \widehat{\mathbf{A}}_{i}^{T}\left[\mathbf{Y}_{i}^{*}-E y_{i-1}(N)\right], \\
& u_{i}=u_{i-1}+\mathbf{g}^{T} \Delta \mathbf{U}_{i},
\end{aligned}
$$

where $\varepsilon$ and $M$ are positive constants; $\widehat{\mathbf{A}}_{i}$ and $\widehat{\theta}_{i+j}$ are the estimated values of $\mathbf{A}_{i}(k)$ and $\theta_{i+j}, j=1, \ldots,\left(N_{y}-1\right)$, respectively; $\lambda>0, \mu>0, \eta \in(0,1], \delta \in(0,1]$.

Remark 5. The initial value of partial derivative estimation is generally set to be $\hat{\theta}_{1}>0$, since $\theta_{i}>0$ holds for many practical industrial systems, such as temperature control system, pressure control system. The proposed DDPTILC scheme has $N_{y}$ parameters to be estimated or predicted by merely using the I/O data of the controlled system.

Remark 6. The DDPTILC approach is proposed for unknown discrete-time nonlinear systems. It requires merely the measured I/O data of the controlled plant for controller design, and thus it is suitable for many practical industrial processes. In contrast, the norm-optimal ILC $[10,11]$ and normoptimal predictive ILC [17] are limited to exactly known linear systems, and the controller should be redesigned by resolving a new complex Riccati equation if there is any little modification or expansion of the controlled plant.

Remark 7. Compared with the optimal terminal iterative learning control in [14], the DDPTILC approach utilizes the predictive terminal output information within a prespecified iteration horizon and thus has better robustness to the iteration-varying desired terminal output signal and iteration-dependent uncertainties.

\section{Convergence Analysis}

In this section, we will discuss the stability and convergence for the DDPTILC scheme (19)-(26).

Theorem 8. If the discrete-time nonlinear system (1), satisfying Assumptions 1-2, is controlled by DDPTILC scheme (19)-(26) for $y_{i}^{*}(N)=y^{*}(N)$, then there exists a constant $\lambda_{\text {min }}>0$, such that the following properties hold for any $\lambda>\lambda_{\min }$.

(a) The tracking error of the system converges; that is, $\lim _{i \rightarrow \infty}\left|e_{i}(N)\right|=\lim _{i \rightarrow \infty}\left|y^{*}(N)-y_{i}(N)\right|=0$.

(b) The system output $y_{i}(N)$ and the control input $u_{i}$ are bounded for all iterations.

Proof. There are three parts for the theorem proof, as shown in the following details.

Firstly, We Will Prove the Boundedness of $\widehat{\theta}_{i}$. If $\left|\widehat{\theta}_{i}\right| \leq \varepsilon$ or $\left|\Delta u_{i-1}\right| \leq \varepsilon$ or $\operatorname{sign}\left(\hat{\theta}_{i}\right) \neq \operatorname{sign}\left(\widehat{\theta}_{1}\right)$, then the boundedness of $\widehat{\theta}_{i}$ is obvious from (20). In the other case, define parameter estimation error as $\widetilde{\theta}_{i}=\widehat{\theta}_{i}-\theta_{i}$. Subtracting $\theta_{i}$ from both sides of the parameter estimation algorithm (19) and using (5) yield

$$
\begin{aligned}
\tilde{\theta}_{i}= & \widetilde{\theta}_{i-1}-\theta_{i}+\theta_{i-1} \\
& +\frac{\eta \Delta u_{i-1}}{\mu+\Delta u_{i-1}^{2}}\left[y_{i-1}(N)-y_{i-2}(N)-\widehat{\theta}_{i-1} \Delta u_{i-1}\right] \\
= & \left(1-\frac{\eta \Delta u_{i-1}^{2}}{\mu+\Delta u_{i-1}^{2}}\right) \tilde{\theta}_{i-1}-\theta_{i}+\theta_{i-1} .
\end{aligned}
$$


From Assumption 2, we have $\left|-\theta_{i}+\theta_{i-1}\right| \leq \alpha_{2}-\alpha_{1}$. Taking absolute value on both sides of (27) yields

$$
\begin{aligned}
\left|\widetilde{\theta}_{i}\right| & \leq\left|\left(1-\frac{\eta \Delta u_{i-1}^{2}}{\mu+\Delta u_{i-1}^{2}}\right)\right|\left|\widetilde{\theta}_{i-1}\right|+\left|-\theta_{i}+\theta_{i-1}\right| \\
& \leq\left|\left(1-\frac{\eta \Delta u_{i-1}^{2}}{\mu+\Delta u_{i-1}^{2}}\right)\right|\left|\widetilde{\theta}_{i-1}\right|+\alpha_{2}-\alpha_{1} .
\end{aligned}
$$

Since $\mu>0$ and $\eta \in(0,1]$, there exists a positive constant $d_{1}$ such that the following inequality holds:

$$
0<1-\frac{\eta \Delta u_{i-1}^{2}}{\mu+\Delta u_{i-1}^{2}} \leq d_{1}<1 .
$$

Noting that (28) and (29), we have

$$
\begin{aligned}
\left|\widetilde{\theta}_{i}\right| & \leq d_{1}\left|\widetilde{\theta}_{i-1}\right|+\alpha_{2}-\alpha_{1} \\
& \leq d_{1}^{2}\left|\widetilde{\theta}_{i-2}\right|+d_{1}\left(\alpha_{2}-\alpha_{1}\right)+\left(\alpha_{2}-\alpha_{1}\right) \\
& \leq \cdots \leq d_{1}^{i-1}\left|\widetilde{\theta}_{1}\right|+\frac{\alpha_{2}-\alpha_{1}}{1-d_{1}} .
\end{aligned}
$$

This means $\widetilde{\theta}_{i}$ is bounded. Since $\theta_{i}$ is bounded, the boundedness of $\hat{\theta}_{i}$ can be guaranteed. The boundedness of the prediction values $\widehat{\theta}_{i+j}, j=1, \ldots,\left(N_{y}-1\right)$, is the direct results of algorithms (21)-(24).

Secondly, We Will Prove Convergence of the Tracking Error. Define terminal tracking error as $e_{i}(N)=y^{*}(N)-y_{i}(N)$. Substituting (5) into tracking error equation and using (25)(26), we have

$$
\begin{aligned}
e_{i}(N)= & y^{*}(N)-y_{i}(N) \\
= & y^{*}(N)-y_{i-1}(N)-\theta_{i} \Delta u_{i} \\
= & y^{*}(N)-y_{i-1}(N)-\theta_{i} \mathbf{g}^{T}\left[\widehat{\mathbf{A}}_{i}^{T} \widehat{\mathbf{A}}_{i}+\lambda \mathbf{I}\right]^{-1} \\
& \times \widehat{\mathbf{A}}_{i}^{T}\left[\mathbf{Y}_{i}^{*}-E y_{i-1}(N)\right] \\
= & y^{*}(N)-y_{i-1}(N)-\theta_{i} \mathbf{g}^{T}\left[\widehat{\mathbf{A}}_{i}^{T} \widehat{\mathbf{A}}_{i}+\lambda \mathbf{I}\right]^{-1} \\
& \times \widehat{\mathbf{A}}_{i}^{T} E\left[y^{*}(N)-y_{i-1}(N)\right] \\
= & \left(1-\theta_{i} \mathbf{g}^{T}\left[\widehat{\mathbf{A}}_{i}^{T} \widehat{\mathbf{A}}_{i}+\lambda \mathbf{I}\right]^{-1} \widehat{\mathbf{A}}_{i}^{T} E\right) e_{i-1}(N) .
\end{aligned}
$$

Taking absolute value on both sides of (31) yields

$$
\left|e_{i}(N)\right| \leq\left|\left(1-\theta_{i} \mathbf{g}^{T}\left[\widehat{\mathbf{A}}_{i}^{T} \widehat{\mathbf{A}}_{i}+\lambda \mathbf{I}\right]^{-1} \widehat{\mathbf{A}}_{i}^{T} E\right)\right|\left|e_{i-1}(N)\right| .
$$

Let $\mathbf{P}=\left[\widehat{\mathbf{A}}_{i}^{T} \widehat{\mathbf{A}}_{i}+\lambda \mathbf{I}\right]$. Since $\widehat{\mathbf{A}}_{i}^{T} \widehat{\mathbf{A}}_{i}$ is a semipositive definite matrix, thus $\mathbf{P}$ and $\mathbf{P}^{-1}$ are positive definite matrix for any $\lambda>0$.
Since $\mathbf{P}^{-1}=\mathbf{P}^{*} / \operatorname{det}(\mathbf{P})$, where $\mathbf{P}^{*}=\left[\begin{array}{ccc}P_{11} & \cdots & P_{N_{y} 1} \\ \vdots & \ddots & \vdots \\ P_{1 N_{y}} & \cdots & P_{N_{y} N_{y}}\end{array}\right]$ is adjoint matrix of $\mathbf{P}$ and $P_{i j}$ is the algebraic cofactor of $\mathbf{P}$, the following equation holds:

$$
\begin{aligned}
\mathbf{g}^{T} & {\left[\widehat{\mathbf{A}}_{i}^{T} \widehat{\mathbf{A}}_{i}+\lambda \mathbf{I}\right]^{-1} \widehat{\mathbf{A}}_{i}^{T} E } \\
& =\mathbf{g}^{T} \mathbf{P}^{-1} \widehat{\mathbf{A}}_{i}^{T} E=\mathbf{g}^{T} \frac{\mathbf{P}^{*}}{\operatorname{det}(\mathbf{P})} \widehat{\mathbf{A}}_{i}^{T} E \\
& =\frac{N_{y} \widehat{\theta}_{i} P_{11}}{\operatorname{det}(\mathbf{P})}+\frac{\left(N_{y}-1\right) \widehat{\theta}_{i+1} P_{21}}{\operatorname{det}(\mathbf{P})}+\cdots+\frac{\widehat{\theta}_{i+N_{y}-1} P_{N_{y} 1}}{\operatorname{det}(\mathbf{P})} .
\end{aligned}
$$

Equation (33) is bounded as $\widehat{\theta}_{i}$ is bounded for all iterations, and its upper bound is a constant independent of $i$.

Since $\mathbf{P}$ is a positive definite matrix, $\operatorname{det}(\mathbf{P})>0$ is a monic polynomial in $\lambda$ of degree $N_{y}, P_{11}>0$ is a monic polynomial in $\lambda$ of degree $N_{y}-1$, and $P_{i 1}\left(i=2,3, \ldots, N_{y}\right)$ is a monic polynomial in $\lambda$ of degree $\left(N_{y}-2\right)$. Thus there exists a constant $\lambda_{\min }>0$, such that (33) has the same positive sign as $P_{11} / \operatorname{det}(\mathbf{P})$ for any $\lambda \geq \lambda_{\text {min }}$. In the sequel, there exists a positive constant $d_{2}$ such that

$$
0<1-\theta_{i} \mathbf{g}^{T}\left[\widehat{\mathbf{A}}_{i}^{T} \widehat{\mathbf{A}}_{i}+\lambda \mathbf{I}\right]^{-1} \widehat{\mathbf{A}}_{i}^{T} E \leq d_{2}<1 .
$$

Combining (32) and (34) gives

$$
\left|e_{i}(N)\right| \leq d_{2}\left|e_{i-1}(N)\right| \leq \cdots \leq d_{2}^{i-1}\left|e_{1}(N)\right| .
$$

Therefore $\lim _{i \rightarrow \infty}\left|e_{i}(N)\right|=0$.

Finally, We Will Prove the Boundedness of the System Output $y_{i}(N)$ and the Control Input $u_{i}$. Since $y^{*}(N)$ is a constant, $y_{i}(N)$ is bounded.

In following, we prove the boundedness of control input sequence. From (25) and (26), we have

$$
\left|\Delta u_{i}\right| \leq\left|\mathbf{g}^{T}\left[\widehat{\mathbf{A}}_{i}^{T} \widehat{\mathbf{A}}_{i}+\lambda \mathbf{I}\right]^{-1} \widehat{\mathbf{A}}_{i}^{T} E\right|\left|e_{i-1}(N)\right| \leq \chi\left|e_{i-1}(N)\right|,
$$

where $\chi$ is a bounded constant since $\widehat{\mathbf{A}}_{i}$ is bounded.

Using (36) recursively, it gives

$$
\begin{aligned}
\left|u_{i}\right| & \leq\left|\Delta u_{i}\right|+\left|\Delta u_{i-1}\right|+\cdots+\left|\Delta u_{2}\right|+\left|u_{1}\right| \\
& \leq \chi\left(\left|e_{i-1}(N)\right|+\left|e_{i-2}(N)\right|+\cdots+\left|e_{1}(N)\right|\right)+|u(1)| \\
& \leq \chi\left(d_{2}^{i-2}\left|e_{1}(N)\right|+\cdots+d_{2}\left|e_{1}(N)\right|\right)+|u(1)| \\
& \leq \chi \frac{d_{2}\left|e_{1}(N)\right|}{1-d_{2}}+|u(1)| .
\end{aligned}
$$

This equation implies that $u_{i}$ is bounded. 


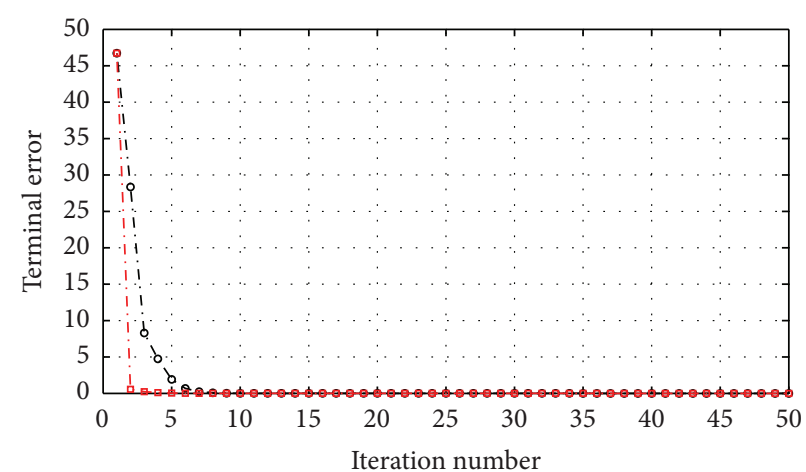

$\multimap-$ TILC

- - DDPTILC

(a) Terminal tracking error

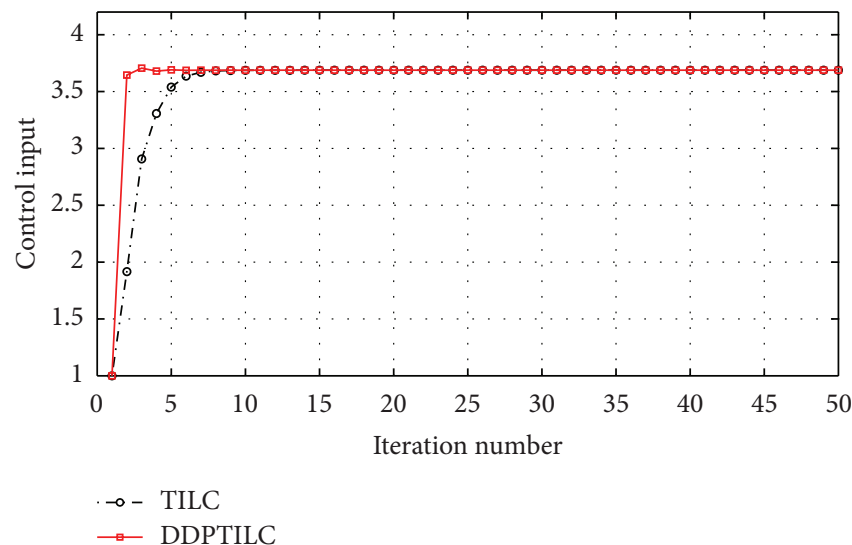

(b) Control input

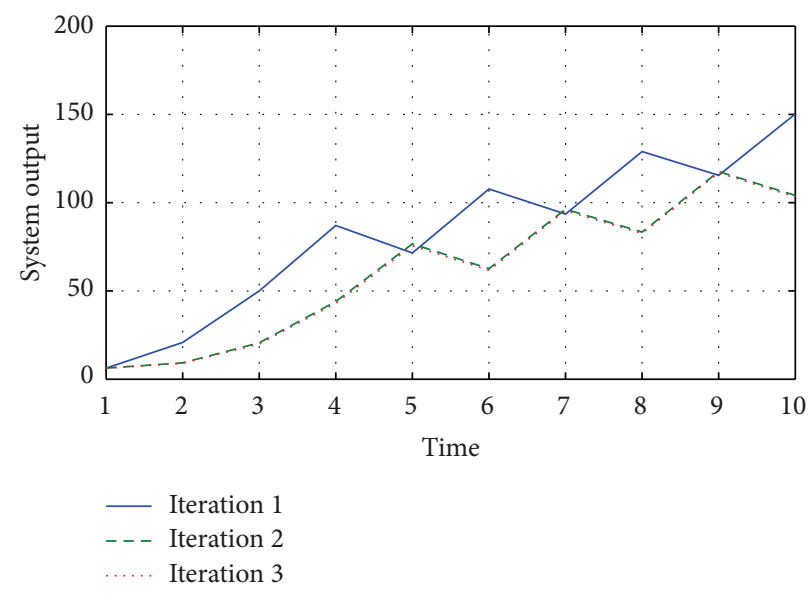

(c) System output with DDPTILC

FIgURE 1: Simulation comparison between TILC and DDPTILC (Case 1).

\section{Simulations}

In this section, the effectiveness of the proposed DDPTILC approach is illustrated through numerical simulations. The mathematical model is assumed to be unavailable for controller design and just serves as the I/O data generator for the train stop system to be controlled.

Consider an ethanol fermentation process [25], whose mechanistic model in the form of differential algebraic equations (DAE) is described as follows [26]:

$$
\begin{gathered}
\frac{d x_{1}}{d t}=C x_{1}-\frac{x_{1}}{x_{4}} u \\
\frac{d x_{2}}{d t}=-10 C x_{1}-\frac{150-x_{2}}{x_{4}} u \\
\frac{d x_{3}}{d t}=D x_{1}-\frac{x_{3}}{x_{4}} u \\
\frac{d x_{4}}{d t}=u \\
y=x_{3}
\end{gathered}
$$

where $x_{1}$ is the cell mass concentration; $x_{2}$ is the substrate concentration; $x_{3}$ is the product concentration; $x_{4}$ is the liquid volume of the reactor; and $C$ and $D$ are two parameters. $x_{4}$ is limited by the $200 \mathrm{~L}$ vessel size. The initial condition is specified as $\left[x_{1}(0), x_{2}(0), x_{3}(0), x_{4}(0)\right]=[1,150,0,10]$. The batch length $t_{f}$ is fixed to be 63 hours and divided into $N=10$ equal stages; that is, sampling time is 6.3 hours. The feed rate into the reactor is used for control and constrained by $0 \leq u \leq 12(1 / h)$. There is no outflow, so the feed rate must be chosen so that the batch volume does not exceed the physical volume of the reactor.

In order to assess the control performance more extensively, three cases are considered in the following simulations. Case 1 is an idea one; the desired output and the system parameters are iteration-invariant. Case 2 is used to verify the good tracking performance of the proposed DDPTILC where the desired output is iteration-varying. Case 3 is used to verify the good tracking performance of the proposed DDPTILC where the system parameters are iteration-varying.

For the purpose of comparison, the data-driven TILC approach proposed in [14] is also simulated on the ethanol 


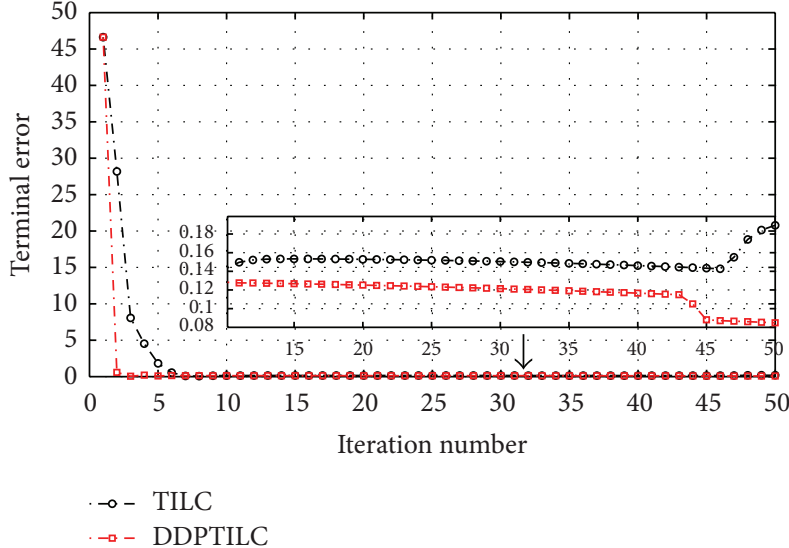

(a) Terminal tracking error

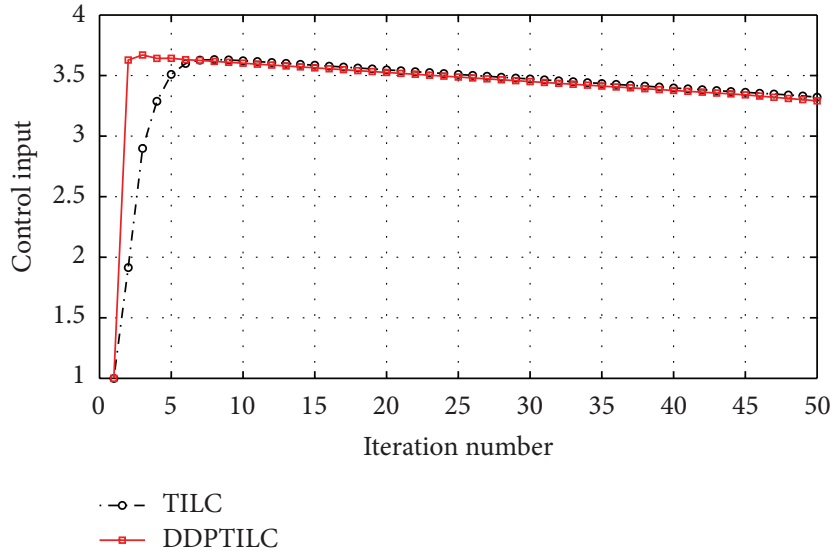

(b) Control input

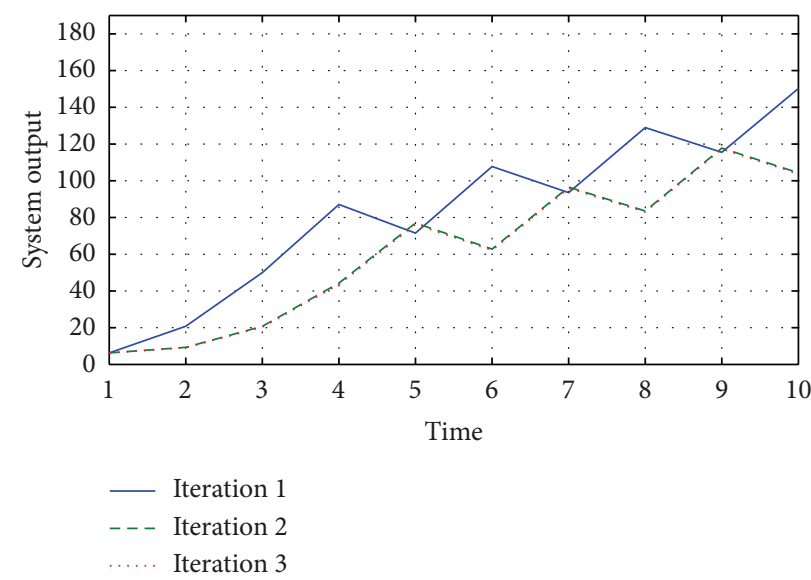

(c) System output with DDPTILC

FIGURE 2: Simulation comparison between TILC and DDPTILC (Case 2).

fermentation process. The TILC approach in [14] is given as follows:

$$
\begin{gathered}
\widehat{\theta}_{i}=\widehat{\theta}_{i-1}+\frac{\eta \Delta u_{i-1}}{\mu+\Delta u_{i-1}^{2}}\left[\Delta y_{i-1}(N)-\widehat{\theta}_{i-1} \Delta u_{i-1}\right], \\
\widehat{\theta}_{i}=\widehat{\theta}_{1}, \quad \text { if }\left|\widehat{\theta}_{i}\right| \leq \varepsilon \text { or }\left|\Delta u_{i-1}\right| \leq \varepsilon \\
\text { or } \operatorname{sign}\left(\widehat{\theta}_{i}\right) \neq \operatorname{sign}\left(\widehat{\theta}_{1}\right), \\
u_{i}=u_{i-1}+\frac{\rho \widehat{\theta}_{i}}{\lambda+\widehat{\theta}_{i}^{2}} e_{i-1}(N) .
\end{gathered}
$$

In general, the tracking error convergence can be guaranteed using the same controller forms and the same controller parameters to different plants provided that the controller parameters are selected in the proper scopes. This is shown in the simulation by using the same controller parameters in the three cases. The parameters of the proposed DDPTILC approach are $N_{y}=3, \theta_{1}=-1, \varepsilon=0.01, M=10, \delta=$ $0.1, \rho=1, \lambda=50, \eta=0.1, \mu=1$ in the following three cases. And the parameters of the TILC approach (39) are $\theta_{1}=-1, \varepsilon=0.01, \rho=1, \lambda=50, \eta=0.1, \mu=1$ in the following three cases.
Case 1. The system parameters and the desired output are iteration-invariant; that is, $C=0.408 x_{2} /\left(1+x_{3} / 16\right)(0.22+$ $\left.x_{2}\right), D=x_{2} /\left(1+x_{3} / 71.5\right)\left(0.44+x_{2}\right)$, and $y^{*}(N)=$ 103.53 were selected from the literature [25]. The simulation results are shown in Figure 1. Figure 1(a) shows the convergence of the terminal tracking error. The horizon is the iteration number and the vertical axis is the absolute values of terminal tracking errors. Figures 1(b) and 1(c) show the profile of control inputs with respect to the iterations and the entire outputs in the first three iterations.

The simulation results show that two TILC approaches guarantee convergence of the terminal tracking error and BIBO stability. It is obvious that the proposed DDPTILC has a faster convergence rate than that of the TILC approach.

Case 2. System parameters are same as that in Case 1. The desired output is iteration-varying; that is, $y^{*}(N)=103.53+$ $10 \sin (i / 100)$, where $i$ denotes the iteration number. The simulation results are shown in Figure 2. Figure 2(a) shows the convergence of the terminal tracking error. Figures 2(b) and 2(c) show the profile of control inputs with respect to the iterations and the entire outputs in the first three iterations. 


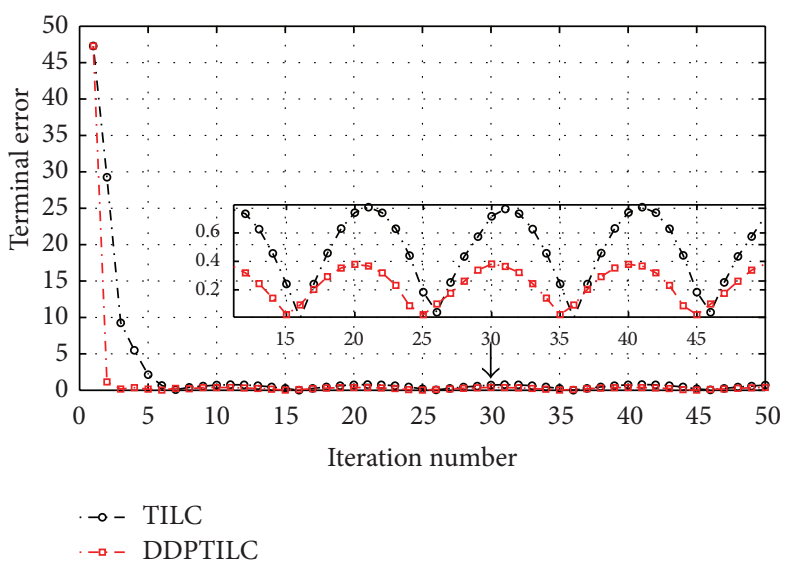

(a) Terminal tracking error

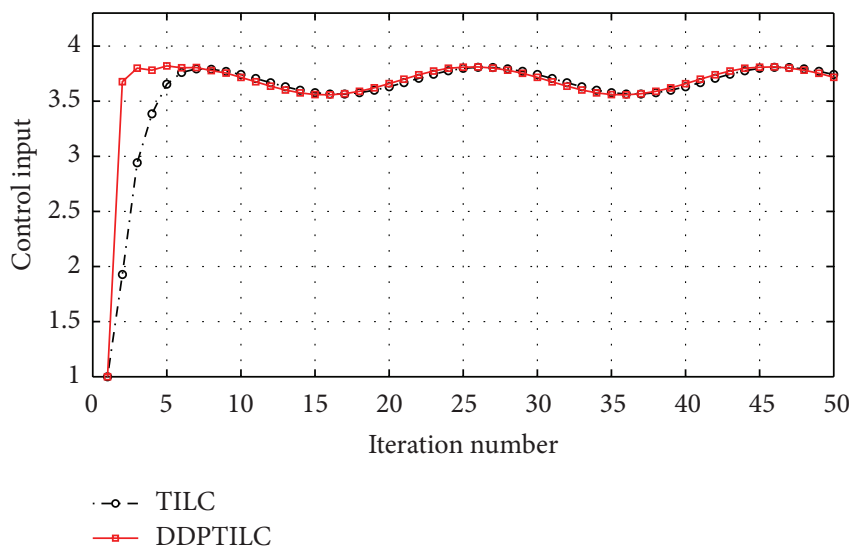

(b) Control input

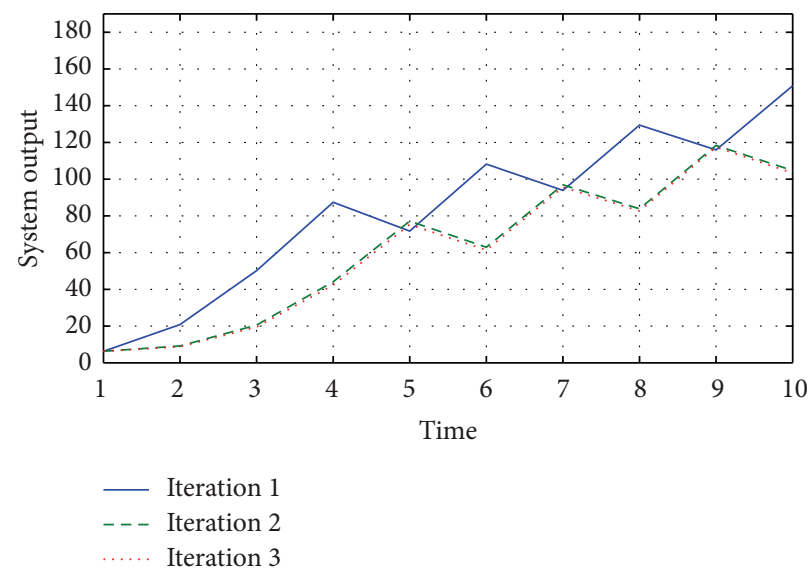

(c) System output with DDPTILC

FIgURE 3: Simulation comparison between TILC and DDPTILC (Case 3).

It is shown that the terminal tracking errors by using two TILC approach converge to a small region when the desired output is iteration-varying. And the DDPTILC approach gives the better terminal tracking performance as shown in Figure 2(a).

Case 3. The desired output is same as that of Case 1. System parameters are iteration-varying; that is, $C=0.408(1+$ $0.01 \sin (2 \pi i / 20)) x_{2} /\left(1+x_{3} / 16\right)\left(0.22+x_{2}\right), D=x_{2} /(1+$ $\left.x_{3} / 71.5\right)\left(0.44(1+0.01 \pi i / 20)+x_{2}\right)$, where $i$ denotes the iteration number. The simulation results are shown in Figure 3. Figure 3(a) shows the convergence of the terminal tracking error. Figures 3(b) and 3(c) show the profile of control inputs with respect to the iterations and the entire outputs in the first three iterations.

It is shown that the terminal tracking errors by using two TILC approach converge to a small region when the system parameters are iteration-varying. And the DDPTILC approach gives the better terminal tracking performance as shown in Figure 3(a).

\section{Conclusions}

This paper presents a new data-driven predictive terminal ILC for a class of discrete-time nonlinear systems only using the terminal output tracking error instead of the whole output trajectory tracking error. The controller design merely depends on the measured I/O data of the plant without requiring plant model information. Rigorous mathematical analysis is developed to illustrate the effectiveness of the proposed approach. Extensive simulation results show the datadriven nature, as well as effectiveness and the applicability, of the proposed predictive terminal ILC further.

\section{Conflict of Interests}

The authors declare that there is no conflict of interests regarding the publication of this paper.

\section{Acknowledgments}

This work is supported by National Natural Science Foundation of China $(61120106009,61374102)$ and the Fundamental Research Funds for the Central Universities (2014JBM005).

\section{References}

[1] H. Ahn, Y. Q. Chen, and K. L. Moore, "Iterative learning control: Brief survey and categorization," IEEE Transactions on Systems, 
Man and Cybernetics C: Applications and Reviews, vol. 37, no. 6, pp. 1099-1121, 2007.

[2] S. Arimoto, S. Kawamura, and F. Miyazaki, "Bettering operation of robots by learning," Journal of Robotic Systems, vol. 1, pp. 123140,1984

[3] J. Xu, "A survey on iterative learning control for nonlinear systems," International Journal of Control, vol. 84, no. 7, pp. 1275-1294, 2011.

[4] J. Xu, Y. Chen, and T. H. Lee, "Terminal iterative learning control with an application to RTPCVD thickness control," Automatica. A Journal of IFAC, the International Federation of Automatic Control, vol. 35, no. 9, pp. 1535-1542, 1999.

[5] G. Gauthier and B. Boulet, "Terminal iterative learning control design with singular value decomposition decoupling for thermoforming ovens," in Proceedings of the American Control Conference (ACC '09), pp. 1640-1645, St. Louis, Mo, USA, June 2009.

[6] Z. Hou, Y. Wang, C. Yin, and T. Tang, "Terminal iterative learning control based station stop control of a train," International Journal of Control, vol. 84, no. 7, pp. 1263-1274, 2011.

[7] T. D. Son, H. Ahn, and K. L. Moore, "Iterative learning control in optimal tracking problems with specified data points," Automatica, vol. 49, no. 5, pp. 1465-1472, 2013.

[8] C. T. Freeman, "Constrained point-to-point iterative learning control with experimental verification," Control Engineering Practice, vol. 20, no. 5, pp. 489-498, 2012.

[9] D. H. Owens, C. T. Freeman, and B. Chu, "Multivariable norm optimal iterative learning control with auxiliary optimisation," International Journal of Control, vol. 86, no. 6, pp. 1026-1045, 2013.

[10] C. T. Freeman and Y. Tan, "Iterative learning control with mixed constraints for point-to-point tracking," IEEE Transactions on Control Systems Technology, vol. 21, no. 3, pp. 604-616, 2013.

[11] T. D. Son and H. Ahn, "Terminal iterative learning control with multiple intermediate pass points," in Proceedings of the American Control Conference (ACC '11), pp. 3651-3656, July 2011.

[12] Z. S. Hou and Z. Wang, "From model-based control to datadriven control: survey, classification and perspective," Information Sciences, vol. 235, pp. 3-35, 2013.

[13] Z. S. Hou and J. X. Xu, "On data-driven control theory: the state of the art and perspective," Acta Automatica Sinica, vol. 35, no. 6, pp. 650-667, 2009.

[14] R. Chi, D. Wang, Z. Hou, and S. Jin, "Data-driven optimal terminal iterative learning control," Journal of Process Control, vol. 22, no. 10, pp. 2026-2037, 2012.

[15] J. Shi, F. Gao, and T. Wu, "Single-cycle and multi-cycle generalized 2D model predictive iterative learning control (2D-GPILC) schemes for batch processes," Journal of Process Control, vol. 17, no. 9, pp. 715-727, 2007.

[16] Y. Wang, D. Zhou, and F. Gao, "Iterative learning model predictive control for multi-phase batch processes," Journal of Process Control, vol. 18, no. 6, pp. 543-557, 2008.

[17] B. Kouvaritakis and M. Cannon, Nonlinear Predictive Control: Theory and Practice, IEE, 2001.

[18] L. Magni, D. M. Raimondo, and F. Allgöwer, Nonlinear Model Predictive Control: Towards New Challenging Applications, Springer, New York, NY, USA, 2009.

[19] Y. Xi, Predictive Control, National Defense Industry, 1993.

[20] R. Chi, Z. Hou, D. Wang, and S. Jin, "An optimal terminal iterative learning control approach for nonlinear discrete-time systems," Control Theory and Applications, vol. 29, no. 8, pp. 1025-1030, 2012.

[21] K. J. Åström and B. Wittenmark, Adaptive Control, New York, NY, USA, Addison-Wesley Longman, 1994.

[22] D. W. Clarke and P. J. Gawthrop, "Self-tuning control," IEE Proceedings, vol. 126, no. 6, pp. 633-640, 1979.

[23] Z. G. Han, "The identification of time-varying parameters in dynamic systems," Acta Automatica Sinica, vol. 10, no. 4, pp. 330-337, 1984.

[24] Z. G. Han, Multi-Level Recursive Method and Its Applications, Science Press, 1989.

[25] Z. Xiong, Y. Xu, J. Zhang, and J. Dong, "Batch-to-batch control of fed-batch processes using control-affine feedforward neural network," Neural Computing and Applications, vol. 17, no. 4, pp. 425-432, 2008.

[26] J. Hong, "Optimal substrate feeding policy for fed batch fermentation with substrate and product inhibition kinetics," Biotechnology and Bioengineering, vol. 28, no. 9, pp. 1421-1431, 1986. 


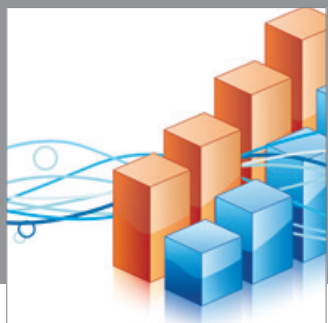

Advances in

Operations Research

mansans

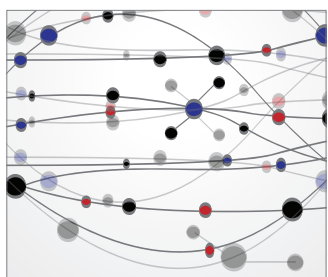

The Scientific World Journal
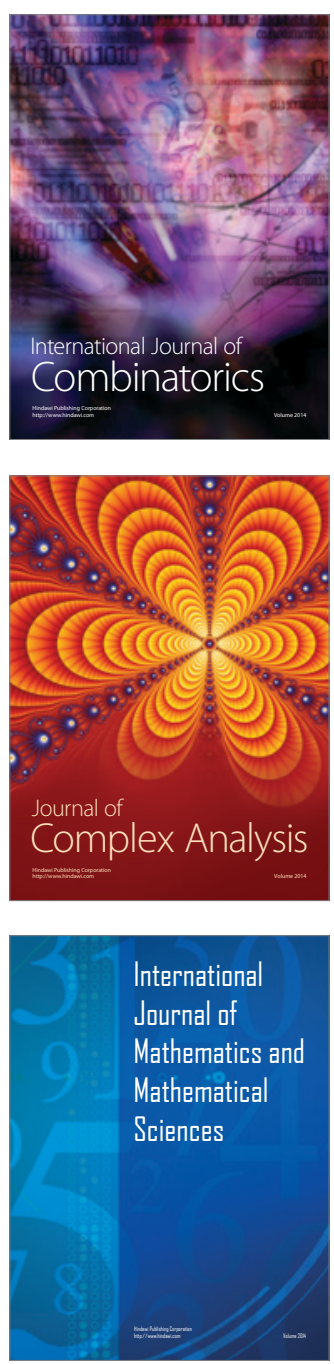
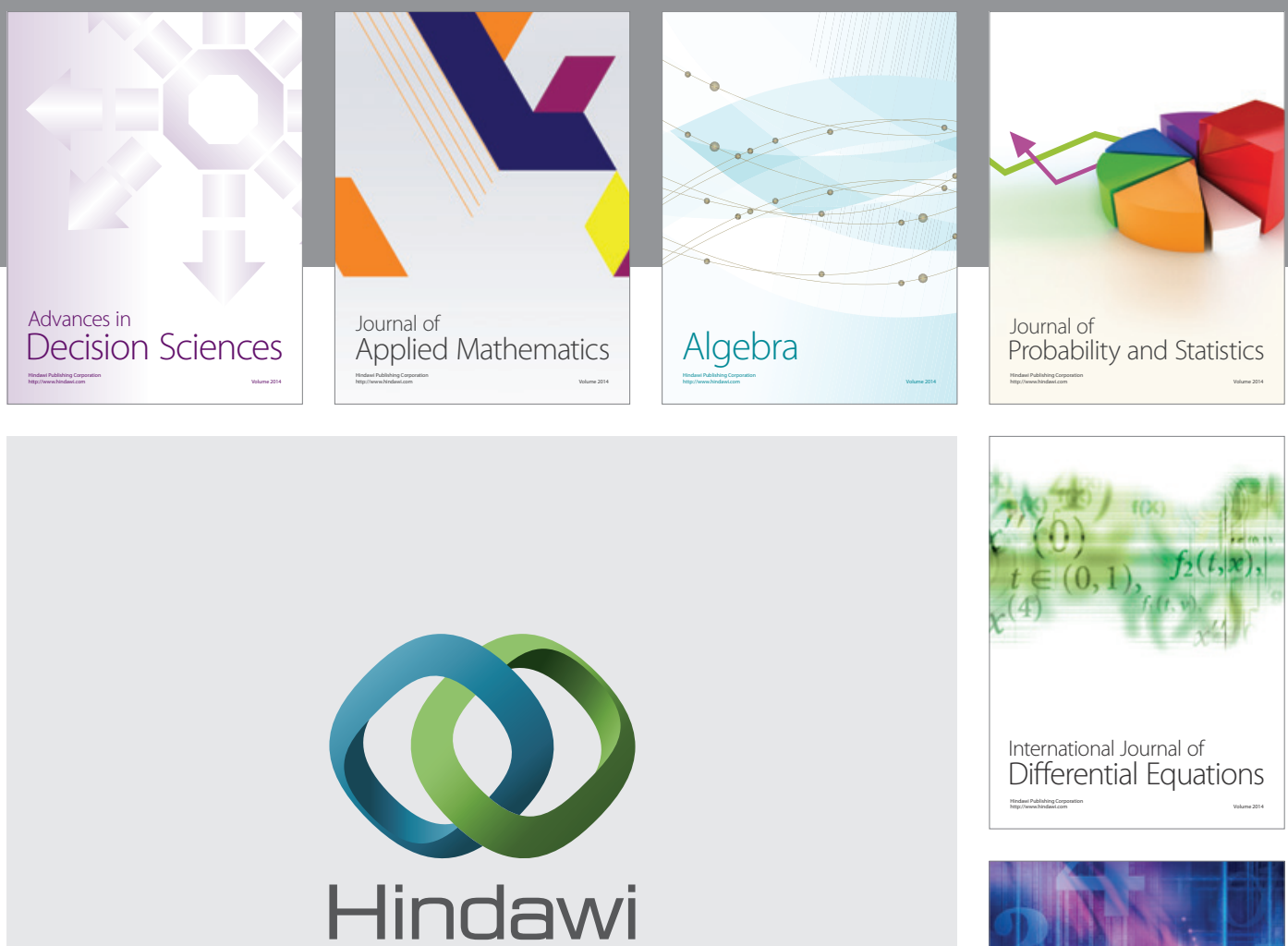

Submit your manuscripts at http://www.hindawi.com
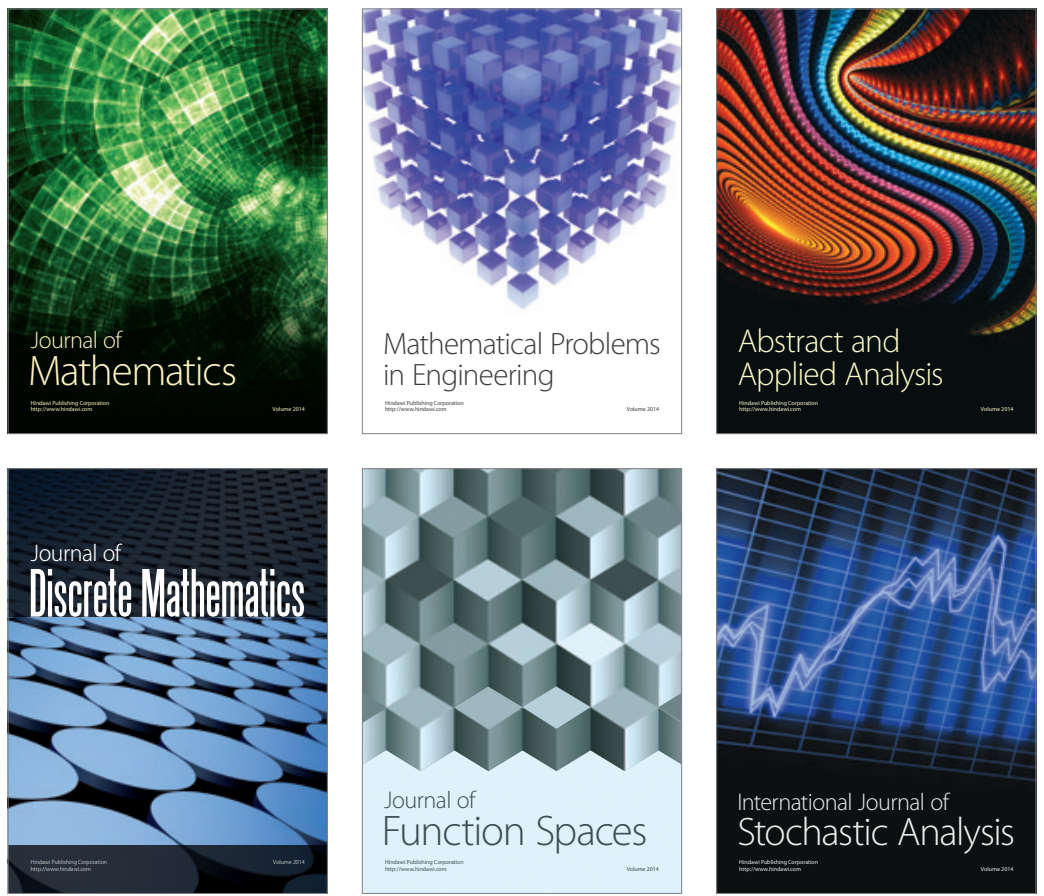

Journal of

Function Spaces

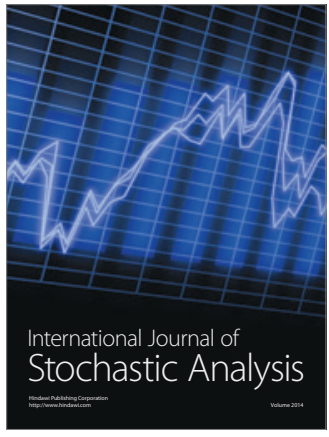

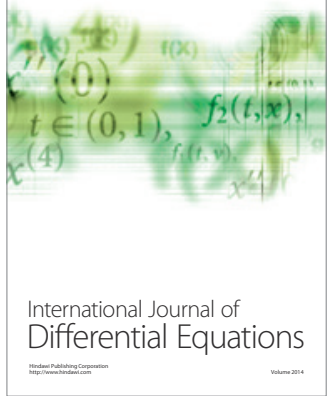
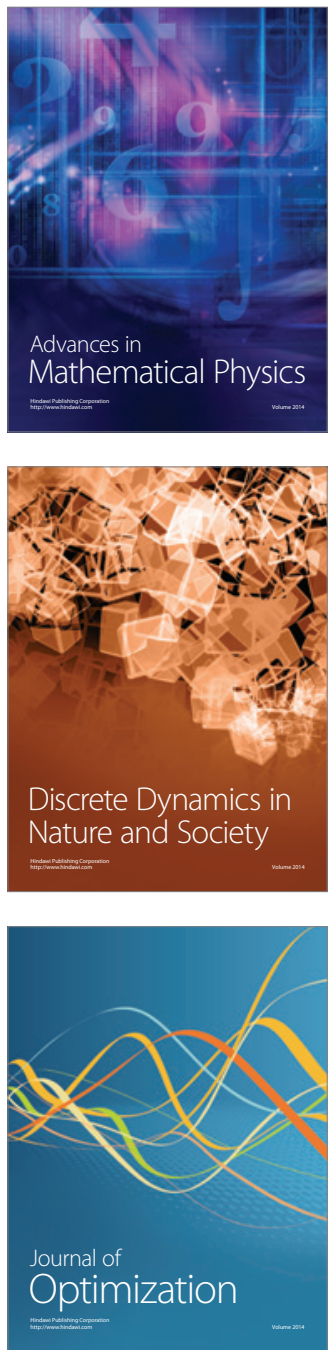